\title{
Plant signaling pathways involved in stomatal movement under drought stress conditions
}

\begin{abstract}
Governed by environmental stimuli and internal signaling cascades, stomatal movement determines water use efficiency and $\mathrm{CO}_{2}$ assimilation for photosynthesis under normal and stressful conditions, most importantly under water scarcity. Manipulation of these signaling pathways is one way to optimize plant resilience/tolerance and performance under constantly changing environment. Oscillations and interactions between the internal and environmental cues make it difficult to determine the consequence of these signals. After drought stress perception, the activated molecules initiate the generation of redox, hormone, and chemical signals, the interactions of which control stomatal responses. Second messengers, proteins, and intermediate chemicals then react with these signaling molecules to positively or negatively transmit these signals through a series of molecular events. This review is aimed to discuss the step-by-step scheme of these signaling pathways to provide insight into these molecular events and to incentivize further studies on their unknown aspects for improved stomatal responses under various environmental conditions, particularly stress conditions, and specifically drought stress.
\end{abstract}

Keywords: plant signaling pathways, stomatal responses, drought stress, molecules, hormonal and chemical signals, ABA, anion and cation channels roles in stomatal movement

\author{
Volume 8 Issue 3 - 2018
}

\author{
Omid Askari-Khorsgani,' Francisco B Flores, ${ }^{2}$ \\ Mohammad Pessarakli ${ }^{3}$ \\ 'Young Researchers and Elite Club, Department of Horticulture, \\ College of Agriculture and Natural Resources, Isfahan \\ (Khorasgan) Branch, Islamic Azad University, Iran \\ ${ }^{2}$ CSIC Tenured Scientist in Plant Biology, Department of Stress \\ Biology and Plant Pathology, Spain \\ ${ }^{3}$ Professor, School of Plant Sciences, College of Agriculture and \\ Life Sciences, The University of Arizona, Tucson, USA
}

\begin{abstract}
Correspondence: Mohammad Pessarakli, Editor-in-Chief, Advances in Plants \& Agriculture Research, Professor, School of Plant Sciences, College of Agriculture and Life Sciences, The University of Arizona, Tucson, AZ 8572I, USA, Email pessarak@email.arizona.edu
\end{abstract}

Received: April 09, 2018| Published: June 18, 2018

\section{Introduction}

Stomatal movement plays a critical role in water hydraulic conductivity and gas exchange capacity, which subsequently control nutrients uptake, temperature adjustment, $\mathrm{CO}_{2}$ assimilation, and thus growth, survival, and tolerance under water stress conditions MartinSt Paul et al. ${ }^{1}$ Opening and closing of stomata is caused by a variety of stimuli such as light/dark, ozone, $\mathrm{CO}_{2}$ concentration, humidity and multiple signaling networks generated by phytohormonrs (abscisic acid (ABA), ethylene (ETH), salicylic acid (SA), methyl jasmonates (MeJA), cytokinin (CK), auxin (AUX), brassinosteroid (BR)), redox $\left(\mathrm{H}_{2} \mathrm{O}_{2}\right.$ and other reactive oxygen species (ROS), nitrous oxide (NO), chemical signals [H2O2and other reactive oxygen species (ROS) like nitrous oxide (NO)], enzymes (phospholipases $\mathrm{C}$ and $\mathrm{D}$ and their products; inositol 1,4,5-trisphosphate ( $\mathrm{InsP}_{3}$ ), phosphatidyl-Ins $\mathrm{P}_{3}$, inositol-6-phosphate; farnesyltransferases, gamma-aminobutyric acid (GABA), protein kinases such as calmodulin-like20 (CaML20)), bioactive gas hydrogen sulfide $\left(\mathrm{H}_{2} \mathrm{~S}\right)$, bioactive lipids (sphingosine-1 phosphate, phospholipids derivatives, sphingolipids, fatty acids like some polyunsaturated ones, linolenic and arachidonic acid), etc. Blackman, Bhatia, Joon-Sang, Allen, Tanaka, Vahisalu, Xia, Misra, Marowa, Nazareno, Jin, Eisenach, Wu, et al. ${ }^{2-14}$ In addition to the mentioned molecular signals, carbohydrate and polyamine metabolism Misra et al., ${ }^{9}$ and interactions between protein kinases such as $\mathrm{Ca}^{2+}$-sensors and Sucrose non-fermenting Related protein Kinases (SnRK2s) may positively or negatively regulate ABA-dependent or independent stomatal movements Aubert Kulik, Bucholc, Kim, Maierhofer, Yoshida, Malcheska et al. ${ }^{15-21}$, Besides hormones crosstalk, the combination of different stresses, duration, severity, repetition, Barrero, Berger, Zandkarimi et al., ${ }^{22,23,24}$ circadian rhythms Grundy, Lee et al. ${ }^{25,26}$ and sudden loss of hydraulic conductance that cause embolism Tombesi et al., ${ }^{27}$ make their interpretations more difficult. Therefore, decoding these interactions and the process of embolism removal can be regarded as effective strategies to improve plant tolerance, recovery, and performance under stressful conditions. In 1974, Levitt ${ }^{28}$ reported that explosion of information on stomatal action led to a rejection of the old, classical theory of the mechanism, proposed by Scarth ${ }^{29}$ in 1932 and its replacement by Fujino ${ }^{30}$ report in 1959 , describing the concept of active $\mathrm{K}^{+}$ion transport. Many of the new facts, however, do not seem to fit into either concept, and neither provide a sufficiently detailed, step-by-step scheme to account for all the known facts. Therefore, Levitt ${ }^{28}$ provided enough information to support his report that is still valid and this information is now available for the proposal of a new scheme which embodies the best features of each of the above concepts and eliminates the inadequacies of each proposal on stomatal movement. The molecular mechanisms underlying this set of signal cross talks and interactions have not been fully discovered yet. Hence, this review article is aimed to provide more insights on this concept by reporting the relevant available literature on this subject.

\section{Hormones crosstalk regulates stomatal movement during day/night cycle and under drought stress conditions}

After perception of drought stress, abscisic acid (ABA) is synthesized in the plastids and transported into cytosols $\mathrm{Xu}$ et $\mathrm{al} .{ }^{31}$ in roots (root tips, vascular bundles of roots) and stem vascular systems (phloem companion cells and xylem parenchyma cells next to the phloem sieve cells and xylem vessels, respectively; leaf veins and florescence stems), as well as leaf mesophyll, Malcheska, Koiwai et al., ${ }^{21,32}$ leaf cuticle, Wang et al., ${ }^{33}$ and guard cells Koiwai et al., ${ }^{32}$ Methyl jasmonate (MeJA), ABA and ethylene (ETH) are effectors of stomatal closure, however, their effects vary depending 
on their concentrations and combinations so that respectively $\mathrm{ABA}+\mathrm{ETH}+\mathrm{MeJA}>\mathrm{MeJA}+\mathrm{ABA}$ or $\mathrm{MeJA}+\mathrm{ETH}>\mathrm{ABA}$ or $\mathrm{ETH}>\mathrm{ABA}+\mathrm{ETH}$ exert stronger effects to induce stomatal closure Nazareno \& Hernandez. ${ }^{11}$ Increased cytokinin $(\mathrm{CK})\left(>10^{-1} \mathrm{~mol} \mathrm{~m}^{-3}\right)$ to $\mathrm{ABA}\left(<10^{-1} \mathrm{~mol} \mathrm{~m}^{-3}\right)$ ratio contributes to reversal ABA-stimulated stomatal opening Blackman \& Davies. ${ }^{2}$ Tanaka and co workers study on Arabidopsis demonstrated that CK and auxin (AUX) hormones can act as negative ABA regulators, inhibiting ABA-induced stomatal closure by promoting ETH biosynthesis Tanaka et al. ${ }^{6}$

However, because He et al ${ }^{34}$ study showed that ETH can produce $\mathrm{H}_{2} \mathrm{O}_{2}$ in light condition to stimulate stomatal closure, the effects of CK and AUX on inhibiting ABA-ETH-induced stomatal closure could possibly be attributed to their effects on hydrogen peroxide $\left(\mathrm{H}_{2} \mathrm{O}_{2}\right)$ removal by $\mathrm{CK}$ and deactivation of nicotinamide adenine dinucleotide phosphate (NADPH) oxidase to inhibit $\mathrm{H}_{2} \mathrm{O}_{2}$ production by AUX as reported by Song et al., ${ }^{35}$ during the day, so not enhancing ETH production. Accordingly, during the day, CK and AUX counteract the effects of ABA and ETH to inhibit stomatal closure, while at night they cooperate with ETH at the requested concentrations for $\mathrm{H}_{2} \mathrm{O}_{2}$ removal to facilitate stomatal opening. In either case, CKs and AUXs induce inhibitory effects on stomatal closure, He, Song et al. ${ }^{34,35}$ Ethylene (ETH) plays a dual function that mediates ultraviolet band (UV-B)-induced stomatal closure via peroxidase-dependent $\mathrm{H}_{2} \mathrm{O}_{2}$ generation during the day $\mathrm{He}$ et al. ${ }^{34}$ and facilitates stomatal opening by $\mathrm{H}_{2} \mathrm{O}_{2}$ removal in darkness Song et al. ${ }^{36}$ The effects of ETH on stomatal closure or opening depends on ETH concentration and its interactions with other phytohormonrs such as CK, AUX, Tanaka et al., ${ }^{6}$ and brassinosteroid (BR) that result in the generation of different amounts of $\mathrm{H}_{2} \mathrm{O}_{2}$ and nitrous oxide (NO) Shi et al. ${ }^{37}$ Like ETH, 24-epibrassinolide (EBR), a natural form of BR, stimulates both stomatal closure and opening based on its concentration-dependent effect on hydrogen peroxide $\left(\mathrm{H}_{2} \mathrm{O}_{2}\right)$ homeostasis and $\mathrm{NO}$ Xia, Shi et al. ${ }^{8,37}$ BR and EBR stimulate ETH synthesis, thereby activating Go protein (G protein $\alpha$-subunit), and ETH cooperatively with activated$\mathrm{G} \alpha$ protein stimulate AtrbohF-mediated $\mathrm{H}_{2} \mathrm{O}_{2}$ and subsequent Nial (nitrate reductase 1)-catalyzed $\mathrm{NO}$ production and consequently induce stomatal closure Shi et al. ${ }^{37}$ In contrast to CK and AUX effects, $\mathrm{SA}$ and also tannic acid act as a positive regulators of $\mathrm{ABA}$, promoting stomatal closure by stimulating ABA biosynthesis and possibly reducing the activity of phosphatase and ATPase enzymes Bhatia, Hao et al..$^{3,38}$

Mitogen-activated protein kinase (MAPK) phosphatases might positively or negatively regulate stomatal closure depending on the interactions between MAPKs and the effects of their isoform on $\mathrm{H}_{2} \mathrm{O}_{2}$ and NO generation Li \& Wang. ${ }^{39}$ Thus, understanding the hormone crosstalk, the influence of hormones on MAPK isoforms, and MAPKs interactions on redox status is required for accurate interpretation of their effects on stomata movement. Drought stress induces ionic $\left(\mathrm{SO}_{4}^{2-}\right)$, redox, and hormonal (brassinosteroid (BR), salicylic acid (SA)) signals and subsequently second messengers $\left(\mathrm{Ca}^{2+}, \mathrm{InsP}_{3}\right)$ to regulate the transcription of important genes like the one encoding 9-cis-epoxycarotenoid dioxygenase (NCED), a key enzyme in ABA biosynthesis, Xia, Malcheska, Hao Estrada-Melo et al. ${ }^{8,21,38,40}$ In return, $\mathrm{ABA}$ activates anion and cation channels and regulates redox homeostasis-induced by NADPH oxidase activity. The NADPH oxidase-generated redox signals induce biosynthesis of $\mathrm{ABA}$ and consequently an ABA self-regulatory system is generated, which plays a fundamental role in regulation of many aspects of cellular processes and stress responses such as stomatal closure (Figure 1) Nazareno \& Hernandez, Malcheska, Harb, Fujita, Castillo, et al. ${ }^{11,21,41-}$ ${ }^{43}$ During the day, methyl jasmonate (MeJA) interacts with ethylene (ETH) Nazareno \& Hernandez $z^{11}$ or ABA to promote stomatal closure (Figure 1) Munemasa et al. ${ }^{44}$ In Arabidopsis, ABA-signaling activates $\mathrm{Ca}^{2+}$-dependent protein kinases (CDPKs), $\mathrm{Ca}^{2+}$ protein kinase (CPK), CPK3, CPK6, CPK4, and CPK11 to phosphorylates ABA-binding factor $(\mathrm{ABF})$ transcription factor (TFs), regulating gene expression involved in MeJA biosynthesis Munemasa, Li et al ${ }^{44,45} \mathrm{ABA}$-activated form of CPK6 induces genes expression encoding both MeJA and ETH biosynthesis, which, in return, activate NADPH oxidases to generate redox signaling. $\mathrm{H}_{2} \mathrm{O}_{2}$ production is mediated by ETH- or ABAmediated NADPH oxidases activity, while MeJA directly induces NO, and ROS generation (Figure 1) Munemasa, Li, Hossain et al. ${ }^{44-46}$ Unlike ABA that induces $\mathrm{H}_{2} \mathrm{O}_{2}$ generation by activating open stomata 1 (OST1) and subsequently activation of NADPH oxidases, MeJA is capable of inducing ROS and NO possibly through activation of NADPH oxidases by cyclic adenosine 5'-diphosphoribose (cADPR), the molecular mechanisms of which remain unknown Hossain et al. ${ }^{46}$ MeJA-induced cADPR function downstream of ABA-induced cyclic guanosine monophosphate (cGMP) to activate NADPH oxidases and subsequently generate ROS signaling (Figure 1). Reactive oxygen species (ROS) react with nitrous oxide (NO) to form reactive $\mathrm{N}$ species such as peroxynitrite $\left(\mathrm{ONOO}^{-}\right)$, which reacts with cyclic guanosine monophosphate (cGMP) to produce 8-nitro-cGMP and activates cyclic nucleotide-gated channel2 (CNGC2), to elevate cytosolic free $\mathrm{Ca}^{2+}\left[\mathrm{Ca}^{2+}\right]_{\text {cyt }}$ in association with MeJA-induced cyclic adenosine 5'-diphosphoribose (cADPR), stimulating ABA-MeJA induced stomatal closure Hossain et al. ${ }^{46} \mathrm{Ca}^{2+}$ channels are activated by 3',5'- cyclic adenosine monophosphate (cAMP), but not cGMP, the molecular mechanism of which remains elusive Hossain et al. ${ }^{46}$ MeJA/cADPR-generated ROS signaling respectively target CPK6 and $\mathrm{Ca}^{2+}$ channels that elevate $\left[\mathrm{Ca}^{2+}\right]_{\text {cyt }}$, and S-type anion channels to synergistically share ABA-MeJA-induced stomatal closure, but not in the absence of ABA (Figure 1) Hossain, Munemasa et al. ${ }^{46,47}$ $\mathrm{Ca}^{2+}$-binding proteins (e.g. CDPKs and $\mathrm{CaMs}$ ) have the potential to stimulate MeJA-mediated NO and ROS production. Munemasa and coworkers showed that CPK6 was not responsible for ROS and NO generation in Arabidopsis Munemasa et al. ${ }^{44}$ ABA-CPK6 interaction mediates MeJA biosynthesis and then MeJA acts as a positive regulator of ABA to facilitate stomatal closure by:

a) Inducing the expression of genes encoding $\mathrm{ABA}$ biosynthesis, e.g., AtNCED3, or

b) Generating ROS and NO in an unknown way, which subsequently cooperatively with ABA restore CPK6 activation to open $\mathrm{Ca}^{2+}$ and S-type anion channels (Figure 1) Munemasa, Hossai, Hossain, Daszkowska-Golec \& Szarejko et al. ${ }^{44,46-49}$ The activation of $\mathrm{Ca}^{2+}$ channels is necessary for elevation of $\left[\mathrm{Ca}^{2+}\right]_{\text {cyt }}$, while the activation of $\mathrm{Ca}^{2+}$ sensor calmodulin $(\mathrm{CaM})$ is necessary for MeJA-induced stomatal closure Munemasa et al. ${ }^{44}$ Thus, this review concludes that in the absence of ABA, when $\mathrm{Ca}^{2+}$ channels and $\mathrm{Ca}^{2+}$ sensor $\mathrm{CaMs}$ are not activated by ABA-induced $\left[\mathrm{Ca}^{2+}\right]_{\mathrm{cyt}}$ elevation, the existence of stress-induced ETH is required Nazareno \& Hernandez ${ }^{11}$ to function downstream of CPK6 signaling and/or to cooperatively with MeJA induce stomatal closure (Figure 1). 


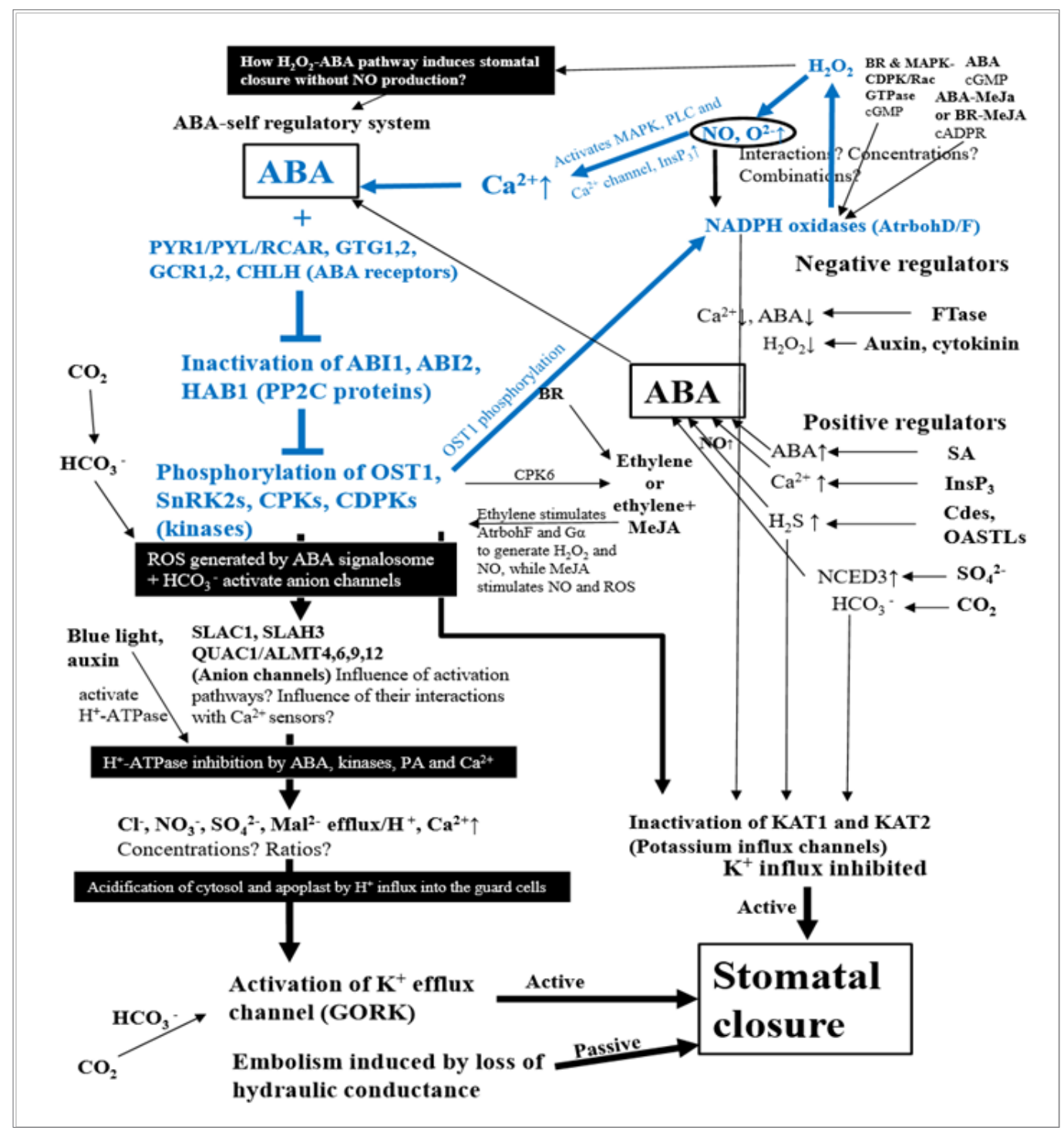

Figure I Metabolic and signaling pathways involved in stomatal closure.

Roles of ABA in stomatal movement in response to drought stress

ABA perception relies on its binding to various receptors localized in cytosol, nucleus, chloroplasts, plasma membrane $\mathrm{Xu}$, Castillo, Zhu, Li et al. ${ }^{31,43,50,51}$ Golgi bodies, and endoplasmic reticulum Jaffé et al. ${ }^{52}$ Heterotrimeric guanine $(\mathrm{G})$ nucleotide-binding protein-coupled receptors (GCRs) consisting of $\alpha, \beta$, and $\gamma$ subunits function together in the $G$ protein signaling pathway to regulate ABA responses in stomatal movement. AtGTG1 (Arabidopsis thalian GCR type Grotein 1), AtGTG2, GCR1, and GCR2 interact with the G protein alpha subunit GPA1 (heterotrimeric Guanine nucleotide-binding Protein Alpha subunit 1) to mediate responses to multiple stresses, including drought, by regulating ABA signaling and the activity of many enzymes for stomatal movement. For instance, regulation of phosphatidylinositol-phospholipase C (PI-PLC) and phospholipase D (PLD) can be mediated by GCR1-GPA1 interaction Jaffé, Klingler, Apone, Pandey \& Assmann, Urano, Chakraborty et al..$^{52-57}$ In this context, understanding the influence of different $\mathrm{ABA}$ receptors, their interactions, and their target molecules are of critical importance for improved, resilient ABA-mediated signal processing and, thereby, stomatal conductance as occurs under optimal condition.

Rapidly upregulated by water stress, $A B A$ Insensitive 1 ( $A B I 1)$, $A B A$ Insensitive 2 ( $A B I 2)$ and Hypersensitive to $A B A 1$ (HAB1) genes encode clade A protein phosphatase $2 \mathrm{Cs}$ (PP2Cs), acting as negative regulators of $\mathrm{ABA}$ responses. Perception of $\mathrm{ABA}$ by its receptors (pyrabactin resistance1/pyrabactin resistance 1-like/regulatory components of ABA receptors; PYR/PYL/RCAR) sequesters ABA and form complexes that inactivate PP2Cs in an ABA-dependent manner Nazareno \& Hernandez, Malcheska, Harb, Fujita, Castillo, Geiger, et al. ${ }^{11,21,41-43,58,59}$ Inactivation of PP2Cs by the ABA-PYLs initiates the ABA-dependent signal transduction pathway where SnRK2type protein kinases SnRK2.2, SnRK2.3 and particularly SnRK2.6/ open stomata 1 (OST1) are released to positively transmit ABA signalosome Zhu. ${ }^{60} \mathrm{ABA}$ signalosome phosphorylates SnRK2s and 
subsequently activates a series of ion channels and ABA-responsive element binding protein (AREB)/ABA-binding factor (ABF) basic leucine zipper (bZIP) transcription factors (TFs) to regulate ABAmediated stress responses such as stomatal movement Nazareno \& Hernandez, Malcheska, Harb, Fujita, Castillo, Li, et al. ${ }^{11,21,41-43,51,59}$ ABA-induced $\mathrm{Ca}^{2+}$-independent OST1 phosphorylates ABF3 to create a 14-3-3 binding motif required for the long-term regulation of gene expression, Sirichandra et al. ${ }^{61}$ suggesting that regulation of ABF3 and 14-3-3 binding sites might be good candidates for the homeostasis of ABA signal to maintain optimal stomatal conductance. In this context, as the final step and perhaps the most effective approach to regulate stomatal movement, the interaction between anion channel regulators such as OST1-CDPKs, OST1-SnRK2s, and OST1- $\mathrm{Ca}^{2+}$-sensor proteins have the potential to improve the stomatal movement under both normal and stressful conditions. Regarding the activation of ion channels, ABA-induced OST1:YFPNT (fusion of the N-terminal half of the yellow fluorescent protein (YFP) to the $\mathrm{C}$ terminus of OST1) phosphorylation activates slow anion channel 1(SLAC1) Maierhofer, Guzel Deger et al., ${ }^{19,62}$ and quickly activating anion channel1/aluminium-activated anion channels (QUAC1/ ALMT12) Eisenach et al., ${ }^{13}$ allowing the release of anions, as well as facilitating potassium efflux by stimulating potassium efflux channels and inhibiting the activity of inward potassium channels, respectively Maierhofer, Guzel Deger et al. 19,62 (Figure 1). However, OST1 is unable to activate slace homolog 3 (SLAH3) Guzel Deger et al. ${ }^{62}$ Elevation of $\left[\mathrm{Ca}^{2+}\right]_{\text {cyt }}$ activates some of the protein kinases which subsequently activate anion channels; thus, some of them can be regulated via $\mathrm{Ca}^{2+}$-independent pathways.

In this context, it can be surmised that upon osmotic stressinduced $\left[\mathrm{Ca}^{2+}\right]_{\text {cyt }}$ elevation and ABA signal stimulation, ABA receptor-phosphatase RCAR1 interacts with ABI1 to inhibit its phosphorylation and negate its ABA negative regulatory effect and, then, RCAR1/PYL9-ABI1 integrative effect Geiger, Demir et al. ${ }^{58,63}$ activates specific calcium-dependent protein kinases (CPKs) such as CPK3,6,21, and 23, and calcineurin-B-like protein-calcineurin-B-like protein interacting protein kinases (CBL-CIPK) (e.g., CBL1, CBL9 and CIPK23) Maierhofer, Geiger, Guzel Deger et al. ${ }^{19,58,62}$ leading to the activation of both SLAC1 and SLAH3 Maierhofer, Guzel Deger et al. ${ }^{19,62}$ The above mentioned findings suggest that downstream of $\mathrm{NO}_{3}{ }^{-} / \mathrm{Cl}^{-}$signals, regulation of $\mathrm{CPKs}$ and the interaction between CBL-CIPK might be effective to improve stomatal movement by regulating SLAC1 and SLAH3. Differentially induced by drought, salinity and ABA treatment, Responsive to Dehydration 20 (RD20) $\mathrm{TF}$, expressed mostly in non-seed tissues in aerial parts and in guard cells, regulates the expression of different $\mathrm{Ca}^{2+}$-binding caleosin-like proteins $(C L O)$ genes. Consequently, depending on their isoforms, CLO encoded proteins differentially function as positive or negative regulators of $\mathrm{ABA}$, stimulating stomatal closure or facilitating stomatal opening Aubert, Kim et al. ${ }^{15,18}$ Similarly, depending on the isoforms, $\mathrm{Ca}^{2+}$-sensors Aubert, Kim et al., ${ }^{15,18}$ and RING E3 Ubiquitin Ligases Lee, Lim et al., ${ }^{66-68}$ act as positive or negative regulators of ABA to differentially regulate stomatal responses. Overall, stress conditions and the isoforms and interactions between protein kinases, $\mathrm{Ca}^{2+}$-sensor proteins, and E3 Ubiquitin Ligases should be taken into account for determining water use efficiency, plant/crop tolerance and performance under drought stress conditions.

As a positive co-regulator of $\mathrm{ABA}$, root uptake of $\mathrm{SO}_{4}^{2-}$ and its transport in xylem sap can stimulate ABA biosynthesis in guard cells and, in cooperation with $\mathrm{H}_{2} \mathrm{~S}$, promotes stomatal closure Jin,
Malcheska et al. ${ }^{12,21} \mathrm{SO}_{4}{ }^{2-}$ upregulated the expression of NCED3 gene involved in ABA biosynthesis, thereby activating QUAC1/ALMT12 anion channels (Figure 1) Malcheska et al. ${ }^{21} \mathrm{H}_{2} \mathrm{~S}$ participates in stomatal closure by regulation of ABA and $\mathrm{NO}$ production as well as anion and, particularly, $\mathrm{K}^{+}$channels activities. However, because of the contradictory results found up to now more investigations are required in order to fully understand the effects of factors responsible for $\mathrm{SO}_{4}{ }^{-}$and $\mathrm{H}_{2} \mathrm{~S}$ functional diversities and their interactions on stomatal closure by regulation of $\mathrm{ABA}$ and $\mathrm{NO}$ production and activity of anion channels Jin et al. ${ }^{12}$ It has been hypothesized that like farnesyltransferases (FTases), expression of genes encoding enzymes involved in $\mathrm{SO}_{4}^{2-}$ biosynthesis/conversion such as sulfotransferases, phosphoadenosine-5'-phosphosulfate synthase Rath et al. ${ }^{69}$; Mueller $\&$ Shafqat $^{70} 5^{\prime}$-adenylylsulfate reductase, Setya et al., ${ }^{71}$ cysteine desulfhydrases (CDes) and $O$-acetyl-L-serine (thiol) lyases (OASTLs) have the potential to stimulate stomata movement in different ways Jin et al. ${ }^{12}$ Related sulfur metabolites like 3'-phosphoadenosine 5'phosphate play a similar role in stomatal movement Pornsiriwong et al. ${ }^{65}$ Regardless of ABA stimulation, future studies about the $\mathrm{SO}_{4}{ }^{2-}$ and hydrogen sulfide $\left(\mathrm{H}_{2} \mathrm{~S}\right)$ interaction and their intermediary regulators may provide valuable information to show if $\mathrm{SO}_{4}{ }^{-2}$ and $\mathrm{H}_{2} \mathrm{~S}$ have the capacity to directly activate anion channels similar to $\mathrm{HCO}_{3}{ }^{-}$effects and affect stomatal movement.

\section{Roles of anion channels and gated or guard cell outward rectifying outward $\mathrm{K}+$ channel in stomatal movement in response to drought stress}

The influx of $\mathrm{Ca}^{2+}$ from plasma membrane and tonoplast into cytoplasm and anions $\left(\mathrm{Cl}^{-}, \mathrm{NO}_{3}^{-}, \mathrm{SO}_{4}^{2^{-}}, \mathrm{Mal}^{2-}\right)$ efflux from cytoplasm and acidification of the cytosol by $\mathrm{H}^{+}$influx depolarize the plasma membrane of guard cells giving rise to voltage-activation of the $\mathrm{K}^{+}$ release channel gated or guard cell outward rectifying outward $\mathrm{K}^{+}$ (GORK) and deactivation of $\mathrm{K}^{+}$influx channels. Inhibited influx along with enhanced efflux of $\mathrm{K}^{+}$cause guard cell turgor loss and stomatal closure (Figure 1) Marowa, Jin, Malcheska et al. ${ }^{10,12,21}$, Daszkowska-Golec \& Szarejko ${ }^{49}$; Zhang et al. ${ }^{72}$ At low concentration, de-protonated form of malic acid functions as a signaling molecule that activates the vacuolar $\mathrm{Cl}^{-}$inward rectifying channel ALMT9 and causes enhanced $\mathrm{Cl}^{-}$uptake during stomatal opening Eisenach et al. ${ }^{13}$ Phosphorylation by protein kinases or phosphatases regulates the activity of plasma membrane $\mathrm{H}^{+}$-ATPase in guard cells, facilitating stomatal closure Daszkowska-Golec \& Szarejko ${ }^{49}$; Haruta et al. ${ }^{73}$ ABA, ABA-induced $\mathrm{H}_{2} \mathrm{O}_{2}$ Zhang, Planes et al. ${ }^{64,74}$ Phosphatidic acid (PA) Camoni et al..$^{25}$ and $\mathrm{Ca}^{2+}$ Kinoshita et al. ${ }^{76}$ all inhibit the activity of $\mathrm{H}^{+}$-ATPase. GCR1-GPA1 and Phospholipase D $\alpha 1 / \delta$ $(P L D \alpha 1 / P L D \delta)$ genes are involved in plant growth and development processes and responses to abiotic stresses and ABA signaling. They are involved in ABA-induced stomatal closure likely by inducing ROS accumulation and signaling, affecting GORKs activity Pandey \& Harb, Assmann, Chakraborty, Chen, Zhao, Uraji, et al. ${ }^{41,54,57,77-79}$ PLD $\alpha 1$ interact with G protein (GPA1; G $\alpha$ subunit of a heterotrimeric GTP-binding protein) and 14-3-3 protein to regulate cell signaling and metabolism in plants Mishra, Hong et al. ${ }^{80,81}$ While PLD $\alpha 1$ positively transmit ABA signals to induces stomatal closure, PLD $\alpha 1-G P A 1$ interaction suppresses $\mathrm{ABA}$ signaling to facilitate stomatal opening so that ABA signal in gpal and pld 1 l single knockout mutants is not strong enough to inhibit stomatal opening, but GPA1 signal does not affect stomatal closure Mishra et al. ${ }^{87}$ PLD-produced PA induces stomatal closure by firstly binding to the AtrbohD and AtrbohFN-terminus and 
stimulating ROS production, secondly by activating protein kinases (e.g., $\mathrm{H}^{+}$-ATPase, protein kinase $\mathrm{C}$, and MAPKs), and thirdly by suppressing ABI1. PLD 1 1-produced PA interacts with ABI1 at 1:1 ratio and, thereby, inhibits $\mathrm{ABI} 1$ translocation from the cytosol to the nucleus and accordingly stimulates stomatal closure; however, it does not inhibit stomatal opening Mishra, Hong et al. ${ }^{80,81}$ Under salinity and drought stresses, intracellular $\mathrm{Ca}^{2+}\left(\left[\mathrm{Ca}^{2+}\right]_{\mathrm{i}}\right)$ elevation stimulates PLD 1 -mediated PA production which subsequently interacts with SnRKs and regulates activity of vacuolar $\mathrm{H}^{+}$-ATPases. These proton pumps help to maintain the proton gradient that drives $\mathrm{Na}^{+} / \mathrm{H}^{+}$ antiporter activity Hong, Bargmann et al. ${ }^{81,82}$ Increased expression of Arabidopsis $P L D \alpha 1$ in canola promoted stomata movement and, thus, improved water status, biomass accumulation and yield by enhancing drought tolerance Lu et al. ${ }^{83}$ Enhancing tolerance to both drought and salinity, PLD $\alpha 1$ and PLD $\delta$ cooperatively regulate ABA signaling in guard cells, but their functions do not completely overlap Uraji et al. ${ }^{79}$

There are ways of regulating stomatal movement at molecular level independent of $\mathrm{ABA}$, and one of the most interesting of these regulating mechanisms is the one starred by a protein family involved in the posttranscriptional regulation of gene expression known as Glycine-rich RNA-binding proteins (GR-RBPs or GRP) Kwak, Kim et al. ${ }^{84,85}$ GRP2 and GRP4, two members of the eight members of the GR-RBP family found in Arabidopsis, have an impact on seed germination, seedling growth, and stress tolerance of Arabidopsis plants under cold, salt, and dehydration stress conditions Kwak, Kim et al. ${ }^{84,85}$ The functional role and mechanism of action of GR-RBP7 has been investigated in relation to plant response to abiotic stress using a transgenic approach. It has been observed by Histochemical analyses of transgenic plants transformed with a genetic construction with the promoter of $G R$ $R B P 7$ fused with GUS (GR-RBP7PRO:GUS) that GR-RBP7 is highly expressed in guard cells. GRP7 action affects stomatal opening under drought and salinity conditions, and stomatal closing under cold and freezing, indicating that GRP7 exerts a negative effect on the first two types of abiotic stresses and a positive impact on the second one Kim et al. ${ }^{86} G R P 7$ overexpression does not affect stomatal closure induced by $\mathrm{ABA}$, suggesting that stomatal opening and closure regulated by GRP7 occur in an ABA-independent manner. No differences in seed germination or seedling growth were found between wild-type, GRP7-overexpressing transgenic plants and grp7 mutants when treated with ABA further supporting the hypothesis that GRP7 affects stomatal opening and closing in an ABA-independent manner. GRRBPs are involved in post-transcriptional gene expression, so it is conceivable that GR-RBP7 interacts with messenger RNAs (mRNAs) of genes closely related to stomatal movements in guard cells and in this manner it is able to modulate the mRNA processing and folding, with different results on stomatal movement depending on the nature of the stressful condition Kim et al. ${ }^{86}$

\section{Concluding remarks}

Manipulation of the signaling pathways for optimizing plant resilience/tolerance and performance under constantly changing environment was the focus of this review. This review postulated that after drought stress perception, the activated molecules initiate the generation of redox, hormone, and chemical signals, the interactions of which control stomatal responses. It further reported that the second messengers, proteins, and intermediate chemicals then react with these signaling molecules to positively or negatively transmit these signals via a series of molecular events. Moreover, this review discussed the step-by-step scheme of these signalling pathways to provide insight into these molecular events and to incentivize further studies on their unknown aspects for improved stomatal responses under various environmental conditions, particularly stress conditions, and specifically drought stress. Downstream of ABA signal, ET-MeJa homeostasis, OST1-CDPKs, OST1-SnRK2s, and OST1-Ca' ${ }^{2+}$-sensor proteins, CPKs, CBL-CIPK, E3 Ubiquitin Ligases, and PLDs act as the final effectors to regulate anion and cation channels activity and, thereby, stomatal movement. Comparing the variations in signaling pathways under stressful and optimal conditions, regulation and perhaps co-regulation of these effectors require further investigations in order to find the most effective approaches in alleviating the disruptive effects of drought stress on stomatal movement that is probably the most important factor in stomatal gas exchange (water vapor and $\mathrm{CO}_{2}$ efflux and influx), and consequently photosynthesis and plant/crop yield. Revealing the molecular steps of molecular signals, this review provides insights into the crosstalk signals and changes in the mode of actions of signaling molecules that vary depending on the activity of different signaling pathways and the interactions between signaling molecules with the target molecules and, thereby, facilitates the correct interpretation of the signaling events involved in stomatal movement under normal and drought stress conditions. Consequently, the correct interpretation of the signaling events would help the breeders to choose the most effective strategy for engineering drought tolerant plants with optimized stomatal responses.

\section{Acknowledgments}

The authors sincerely appreciate the Editorial Team of the Advances in Plants \& Agriculture Research (APAR), particularly, the Executive/Managing Editor, Dr. Rebecca Miller for diligently handling this manuscript.

\section{Conflict of interest}

The author declares there is no conflict of interest.

\section{References}

1. Martin-StPaul N, Delzon S, Cochard H. Plants resistance to drought relies on early stomata closure. bioRxiv. 2017.

2. Blackman PG, Davies WJ. The Effects of Cytokinins and ABA on stomatal Behaviour of Maize and Commelina. Journal of Experimental Botany. 1983;34(12):1619-1626.

3. Bhatia DS, Jindal V, Malik CP. Effect of salicylic acid and tannic acid on stomatal aperture and some enzyme changes in isolated epidermal peelings of Euphorbia hirta L. Biochemie und Physiologie der Pflanzen. 1986;181(4):261-264.

4. Joon-Sang L. The mechanism of stomatal closing by salicylic acid in Commelina communis L. Journal of Plant Biology. 1998;41(2):97-102.

5. Allen GJ, Murata Y, Chu SP, et al. Hypersensitivity of abscisic acidinduced cytosolic calcium increases in the Arabidopsis farnesyltransferase mutant era1-2. The Plant Cell. 2002;14(7):1649-1662.

6. Tanaka Y, Sano T, Tamaoki M, et al. Cytokinin and auxin inhibit abscisic acid-induced stomatal closure by enhancing ethylene production in Arabidopsis. Journal of Experimental Botany. 2006;57(10):2259-2266.

7. Vahisalu T, Kollist H, Wang Y-F, et al. SLAC1 is required for plant guard cell S-type anion channel function in stomatal signalling. Nature. 2008;452(7186):487-491. 
8. Xia X-J, Gao C-J, Song L-X, et al. Role of $\mathrm{H}_{2} \mathrm{O}_{2}$ dynamics in brassinosteroid-induced stomatal closure and opening in Solanum lycopersicum. Plant, Cell \& Environment. 2014;37(9):2036-2050.

9. Misra BB, Acharya BR, Granot D, et al. The guard cell metabolome: functions in stomatal movement and global food security. Frontiers in Plant Science. 2015;6:334

10. Marowa P, Ding A, Kong Y. Expansins: roles in plant growth and potentia applications in crop improvement. Plant Cell Reports. 2016;35(5):949965

11. Nazareno AL, Hernandez BS. A mathematical model of the interaction of abscisic acid, ethylene and methyl jasmonate on stomatal closure in plants. PloS One. 2017;12(2):e0171065.

12. Jin Z, Wang Z, Ma Q, et al. Hydrogen sulfide mediates ion fluxes inducing stomatal closure in response to drought stress in Arabidopsis thaliana. Plant and Soil. 2017;419(1-2):141-152.

13. Eisenach $\mathrm{C}$, Baetz U, Huck NV, et al. ABA-induced stomatal closure involves ALMT4, a phosphorylation-dependent vacuolar anion channe of Arabidopsis. The Plant Cell. 2017;29(10)2552-2569.

14. Wu X, Qiao Z, Liu H, et al. CML20, an Arabidopsis calmodulin-like protein, negatively regulates guard cell ABA signaling and drought stress tolerance. Frontiers in Plant Science. 2017;8:824

15. Aubert Y, Vile D, Pervent M, et al. RD20, a stress-inducible caleosin, participates in stomatal control, transpiration and drought tolerance in Arabidopsis thaliana. Plant and Cell Physiology. 2010;51(12):19751987.

16. Kulik A, Wawer I, Krzywińska E, et al. SnRK2 protein kinases-key regulators of plant response to abiotic stresses. Omics: A Journal of Integrative Biology. 2011;5(12):859-872.

17. Bucholc M, Ciesielski A, Goch G, et al. SNF1-related protein kinases 2 are negatively regulated by a plant-specific calcium sensor. Journal of Biological Chemistry. 2011;286(5):3429-3441.

18. Kim YY, Jung KW, Yoo KS, et al. A stress-responsive caleosin-like protein, AtCLO4, acts as a negative regulator of ABA responses in Arabidopsis. Plant and Cell Physiology. 2011;52(5):874-884.

19. Maierhofer T, Diekmann M, Offenborn JN, et al. Site- and kinasespecific phosphorylation-mediated activation of SLAC1, a guard cell anion channel stimulated by abscisic acid. Science Signaling 2014;7(342):ra86

20. Yoshida T, Fujita Y, Maruyama K, et al. Four Arabidopsis AREB/ $\mathrm{ABF}$ transcription factors function predominantly in gene expression downstream of SnRK2 kinases in abscisic acid signalling in response to osmotic stress. Plant, Cell \& Environment. 2015;38(1):35-49.

21. Malcheska F, Ahmad A, Batool S, et al. Drought enhanced xylem sap sulfate closes stomata by affecting ALMT12 and guard cell ABA synthesis. Plant Physiology. 2017;174(2):798-814.

22. Barrero JM, Rodriguez PL, Quesada V, et al. Both abscisic acid (ABA) dependent and ABA-independent pathways govern the induction of NCED3, AAO3 and ABA1 in response to salt stress. Plant, Cell \& Environment. 2006;29(10):2000-2008.

23. Berger B, Parent B, Tester M. High-throughput shoot imaging to study drought responses. Journal of Experimental Botany. 2010;61(13):3519 3528 .

24. Zandkarimi H, Ebadi A, Salami SA, et al. Analyzing the expression profile of $\mathrm{AREB} / \mathrm{ABF}$ and $\mathrm{DREB} / \mathrm{CBF}$ genes under drought and salinity stresses in Grape (Vitis vinifera L.). PloS one. 2015;10(7):e0134288.

25. Grundy J, Stoker C, Carré IA. Circadian regulation of abiotic stres tolerance in plants. Frontiers in Plant Science. 2015;6(648)
26. Lee HG, Mas P, Seo PJ. MYB96 shapes the circadian gating of ABA signaling in Arabidopsis. Scientific Reports. 2016;6:(17754):1775117711.

27. Tombesi S, Nardini A, Frioni T, et al. stomatal closure is induced by hydraulic signals and maintained by ABA in drought-stressed grapevine. Scientific Reports. 2015;5:12449.

28. Levitt J. The mechanism of stomatal movement-Once more Protoplasma. 1974;82(1):1-517.

29. Scarth GW. Mechanism of the action of light and other factors on stomatal movement. Plant Physiology. 1932;7(3):481-504.

30. Fujino M. Stomatal movement and active migration of potassium Kagaku. 1959;29:660-5661.

31. Xu Z-Y, Kim DH, Hwang I. ABA homeostasis and signaling involving multiple subcellular compartments and multiple receptors. Plant Cell Reports. 2013;32(6):807-813.

32. Koiwai H, Nakaminami K, Seo M, et al. Tissue-specific localization of an abscisic acid biosynthetic enzyme, AAO3, in Arabidopsis. Plant Physiology. 2004;134(4):1697-1707.

33. Wang Z-Y, Xiong L, Li W, et al. The plant cuticle is required for osmotic stress regulation of abscisic acid biosynthesis and osmotic stress tolerance in Arabidopsis. Plant Cell. 2011;23(5):1971-1984.

34. He J, Yue X, Wang R, et al. Ethylene mediates UV-B-induced stomatal closure via peroxidase-dependent hydrogen peroxide synthesis in Vicia faba L. J Exp Bot. 2011;62(8):2657-2666.

35. Song X-G, She X-P, He J-M, et al. Cytokinin- and auxin-induced stomatal opening involves a decrease in levels of hydrogen peroxide in guard cells of Viciafaba L. Functional Plant Biology. 2006;33(6):573-583.

36. Song XG, She XP, Wang J. Inhibition of darkness-induced stomatal closure by ethylene involves a removal of hydrogen peroxide from guard cells of Viciafaba L. Russian Journal of Plant Physiology. 2012;59(3):372-380.

37. Shi C, Qi C, Ren H, et al. Ethylene mediates brassinosteroid-induced stomatal closure via $\mathrm{G} \alpha$ protein-activated hydrogen peroxide and nitric oxide production in Arabidopsis. Plant J. 2015;82(2):280-301.

38. Hao JH, Wang XL, Dong CJ, et al. Salicylic acid induces stomatal closure by modulating endogenous hormone levels in cucumber cotyledons. Russian Journal of Plant Physiology. 2011;58(5):906.

39. Li FC, Wang J. Mitogen-activated protein kinase phosphatases affect UV-B-induced stomatal closure via controlling NO in guard cells. Plant Physiol. 2016;173(1):760-770.

40. Estrada-Melo AC, Reid MS, Jiang C-Z. Overexpression of an ABA biosynthesis gene using a stress-inducible promoter enhances drought resistance in petunia. Hortic Res. 2015;2(1):15013.

41. Harb A, Krishnan A, Ambavaram MMR, et al. Molecular and physiological analysis of drought stress in Arabidopsis reveals early responses leading to acclimation in plant growth. Plant Physiol. 2010;154(3):1254-1271.

42. Fujita Y, Yoshida T, Yamaguchi-Shinozaki K. Pivotal role of the AREB ABF-SnRK2 pathway in ABRE-mediated transcription in response to osmotic stress in plants. Physiol Plant. 2013;147(1):15-27.

43. Castillo M-C, Lozano-Juste J, González-Guzmán M, et al. Inactivation of PYR/PYL/RCAR ABA receptors by tyrosine nitration may enable rapid inhibition of ABA signaling by nitric oxide in plants. Sci Signal. 2015;8(392):ra89.

44. Munemasa S, Hossain MA, Nakamura Y, et al. The Arabidopsis calcium-dependent protein kinase, CPK6, functions as a positive 
regulator of methyl jasmonate signaling in guard cells. Plant Physiol. 2011;155(1):553-561.

45. Li S, Han X, Yang L, et al. Mitogen-activated protein kinases and calcium-dependent protein kinases are involved in wounding-induced ethylene biosynthesis in Arabidopsis thaliana. Plant, Cell \& Environment. 2017;41(1):134-147.

46. Hossain MA, Ye W, Munemasa S. et al. Cyclic adenosine 5'-diphosphoribose (cADPR) cyclic guanosine 3',5'-monophosphate positively function in $\mathrm{Ca} 2+$ elevation in methyl jasmonate-induced stomatal closure, cADPR is required for methyl jasmonate-induced ROS accumulation NO production in guard cells. Plant Biol (Stuttg). 2014;16(6):1140-1144.

47. Munemasa S, Mori IC, Murata Y. Methyl jasmonate signaling and signal crosstalk between methyl jasmonate and abscisic acid in guard cells. Plant Signal Behav. 2011;6(7):939-941.

48. Hossain MA, Munemasa S, Uraji M, Y. et al. Involvement of endogenous abscisic acid in methyl jasmonate-induced stomatal closure in Arabidopsis. Plant Physiol. 2011;156(1):430-438.

49. Daszkowska-Golec A, Szarejko I. Open or close the gate-stomata action under the control of phytohormones in drought stress conditions. Front Plant Sci. 2013;4:138

50. Zhu M, Monroe J, Suhail Y, et al. Molecular and systems approaches towards drought-tolerant canola crops. New Phytol. 2016;210(4):11691189.

51. Li Z, Waadt R, Schroeder JI. Release of GTP exchange factor mediated down-regulation of abscisic acid signal transduction through ABAinduced rapid degradation of RopGEFs. PLOS Biology. 2016;14 (5):e1002461.

52. Jaffé FW, Freschet G-EC, Valdes BM. G protein-coupled receptor-type $\mathrm{G}$ proteins are required for light-dependent seedling growth and fertility in Arabidopsis. The Plant Cell. 2012;24(9):3649-3668.

53. Apone F, Alyeshmerni N, Wiens K, et al. The G-protein-coupled receptor GCR1 regulates DNA synthesis through activation of phosphatidylinositol-specific phospholipase C. Plant Physiol. 2003;133(2):571-579.

54. Pandey S, Assmann SM. The Arabidopsis putative G protein-coupled receptor GCR1 interacts with the G protein $\alpha$ subunit GPA1 and regulates abscisic acid signaling. Plant Cell. 2004;16(6):1616-1632.

55. Klingler JP, Batelli G, Zhu J-K. ABA receptors: the START of a new paradigm in phytohormone signalling. $J$ Exp Bot. 2010;61(12):31993210 .

56. Urano D, Chen J-G, Botella JR, et al. Heterotrimeric G protein signalling in the plant kingdom. Open Biol. 2013;3(3):1-22.

57. Chakraborty N, Singh N, Kaur K, et al. G-protein signaling components GCR1 and GPA1 mediate responses to multiple abiotic stresses in Arabidopsis. Front Plant Sci. 2015;6(1000).

58. Geiger D, Maierhofer T, AL-Rasheid KAS, et al. Stomatal closure by fast abscisic acid signaling is mediated by the guard cell anion channel SLAH3 and the receptor RCAR1. Sci Signal. 2011;4(173):ra32-ra32.

59. Acharya BR, Jeon BW, Zhang W. Open Stomata 1 (OST1) is limiting in abscisic acid responses of Arabidopsis guard cells. New Phytol. 2013;200(4):1049-1063.

60. Zhu J-K. Abiotic stress signaling and responses in plants. Cell. 2016;167(2):313-324.

61. Sirichandra C, Davanture M, Turk BE, et al. The Arabidopsis ABAactivated kinase OST1 phosphorylates the bZIP transcription factor
ABF3 and creates a 14-3-3 binding site involved in its turnover. PloS One. 2010;5(11):e13935.

62. Guzel Deger A, Scherzer S, Nuhkat M, et al. Guard cell SLAC1-type anion channels mediate flagellin-induced stomatal closure. New Phytol. 2015;208(1):162-173.

63. Demir F, Horntrich C, Blachutzik JO, et al. Arabidopsis nanodomaindelimited ABA signaling pathway regulates the anion channel SLAH3. Proc Natl Acad Sci USA. 2013;110(20):8296-8301.

64. Zhang X, Wang H, Takemiya A, et al. Inhibition of blue light-dependent $\mathrm{H}^{+}$pumping by abscisic acid through hydrogen peroxide-induced dephosphorylation of the plasma membrane $\mathrm{H}+$-ATPase in guard cell protoplasts. Plant Physiol. 2004;136(4):4150-4158.

65. Pornsiriwong W, Estavillo GM, Chan KX, et al. A chloroplast retrograde signal, 3'-phosphoadenosine 5'-phosphate, acts as a secondary messenger in abscisic acid signaling in stomatal closure and germination. ELife. 2017;6:e23361.

66. Lee JH, Kim WT. Regulation of abiotic stress signal transduction by E3 ubiquitin ligases in Arabidopsis. Mol Cells. 2011;31(3):201-208.

67. Kim JH, Kim WT. The Arabidopsis RING E3 ubiquitin ligase AtAIRP3/ LOG2 participates in positive regulation of high-salt and drought stress responses. Plant Physiol. 2013;162(3):1733-1749.

68. Lim CW, Park C, Kim J-H, et al. Pepper CaREL1, a ubiquitin E3 ligase, regulates drought tolerance via the ABA-signalling pathway. Sci Rep. 2017;7(1):477.

69. Rath VL, Verdugo D, Hemmerich S. Sulfotransferase structural biology and inhibitor discovery. Drug Discovery Today. 2004;9(23):1003-1011.

70. Mueller JW, Shafqat N. Adenosine-5'-phosphosulfate-a multifaceted modulator of bifunctional 3'-phospho-adenosine-5'-phosphosulfate synthases and related enzymes. FEBS J. 2013;280(13):3050-3057.

71. Setya A, Murillo M, Leustek T. Sulfate reduction in higher plants: Molecular evidence for a novel 5'-adenylylsulfatereductase. Proc Natl Acad Sci USA. 1996;93(23):13383-13388.

72. Zhang A, Ren H-M, Tan Y-Q, et al. S-type anion channels SLAC1 and SLAH3 function as essential negative regulators of inward $\mathrm{K}+$ channels and stomatal opening in Arabidopsis. The Plant Cell. 2016;52(7186):487491.

73. Haruta M, Gray WM, Sussman MR. Regulation of the plasma membrane proton pump (H+-ATPase) by phosphorylation. Curr Opin Plant Biol. 2015;28:68-75.

74. Planes MD, Niñoles R, Rubio L, et al. A mechanism of growth inhibition by abscisic acid in germinating seeds of Arabidopsis thaliana based on inhibition of plasma membrane $\mathrm{H}+$-ATPase and decreased cytosolic $\mathrm{pH}$, $\mathrm{K}^{+}$, and anions. $J$ Exp Bot. 2015;66(3):813-825.

75. Camoni L, Lucente CD, Pallucca R, et al. Binding of phosphatidic acid to 14-3-3 proteins hampers their ability to activate the plant plasma membrane H+-ATPase. IUBMB Life. 2012;64(8):710-716.

76. Kinoshita T, Nishimura M, Shimazaki K. Cytosolic concentration of $\mathrm{Ca}^{2+}$ regulates the plasma membrane $\mathrm{H}^{+-A T P a s e}$ in guard cells of Fava Bean. Plant Cell. 1995;7(8):1333-1342.

77. Chen J-G, Gao Y, Jones AM. Differential roles of Arabidopsis heterotrimeric G-protein subunits in modulating cell division in roots. Plant Physiology. 2006;141(3):887-897.

78. Zhao Z, Stanley BA, Zhang W, et al. ABA-regulated G protein signaling in Arabidopsis guard cells: a proteomic perspective. J Proteome Res. 2010;9(4):1637-1647. 
79. Uraji M, Katagiri T, Okuma E, et al. Cooperative function of PLD $\delta$ and PLD $\alpha 1$ in abscisic acid-induced stomatal closure in Arabidopsis. Plant Physiol. 2012;159(1):450-460.

80. Mishra G, Zhang W, Deng F, et al. A bifurcating pathway directs abscisic acid effects on stomatal closure and opening in Arabidopsis. Science. 2006;312(5771):264-266.

81. Hong Y, Zhang W, Wang X. Phospholipase D and phosphatidic acid signalling in plant response to drought and salinity. Plant Cell Environ. 2010;33(4):627-635.

82. Bargmann BOR, Laxalt AM, Riet Bt, et al. Multiple PLDs required for high salinity and water deficit tolerance in plants. Plant Cell Physiol. 2009;50(1):78-89.
83. Lu S, Bahn SC, Qu G, et al. Increased expression of phospholipase Dalpha1 in guard cells decreases water loss with improved seed production under drought in Brassica napus. Plant Biotechnology Journal. 2013;11(3):380-389.

84. Kwak KJ, Kim YO, Kang H. Characterization of transgenic Arabidopsis plants overexpressing GR-RBP4 under high salinity, dehydration, or cold stress. J Exp Bot. 2005;56(421):3007-3016.

85. Kim JY, Park SJ, Jang B, et al. Functional characterization of a glycinerich RNA-binding protein 2 in Arabidopsis thaliana under abiotic stress conditions. Plant J. 2007;50(3):439-451.

86. Kim JS, Jung HJ, Lee HJ, et al. Glycine-rich RNA-binding protein 7 affects abiotic stress responses by regulating stomata opening and closing in Arabidopsis thaliana. Plant J. 2008;55(3):455-466. 